International Journal of Child and Adolescent Resilience

Revue internationale de la résilience des enfants et des adolescents

\title{
Développer la résilience chez les jeunes trans et non binaires : un modèle basé sur l'éthique de la reconnaissance d'Axel Honneth
}

\author{
Denise Medico
}

Volume 8, Number 1, 2021

URI: https://id.erudit.org/iderudit/1082073ar

DOI: https://doi.org/10.7202/1082073ar

See table of contents

\section{Publisher(s)}

Canada Research Chair in Interpersonal Traumas and Resilience/Chaire de recherche du Canada sur les traumas interpersonnels et la résilience

\section{ISSN}

2292-1761 (digital)

Explore this journal

Cite this article

Medico, D. (2021). Développer la résilience chez les jeunes trans et non binaires : un modèle basé sur l'éthique de la reconnaissance d'Axel Honneth.

International Journal of Child and Adolescent Resilience / Revue internationale de la résilience des enfants et des adolescents, 8(1), 31-47.

https://doi.org/10.7202/1082073ar

\section{Article abstract}

Notre objectif est de participer à la fondation des bases théoriques et empiriques des approches transaffirmatives pour les jeunes trans et non binaires (JTNB). Nous proposons un modèle dont les bases théoriques s'ancrent dans l'éthique de la reconnaissance d'Axel Honneth et dont les moyens et les outils d'intervention sont développés à partir des écrits sur les facteurs de résilience chez cette population spécifique. La recherche de ces 10 dernières années a montré que les oppressions que les JTNB vivent tout au long de leur développement ont un impact délétère sur leur santé mentale. La question qui se pose aujourd'hui aux intervenant.e.x.s est donc comment favoriser la résilience ? Pour y répondre, nous proposons quatre niveaux d'intervention basés sur les niveaux de reconnaissance sociale d'Axel Honneth et les travaux sur l'intersubjectivité en psychologie : subjectif, intersubjectif, communautaire et légal. En fonction de ces niveaux, les approches transaffirmatives développent des interventions qui ont les objectifs de favoriser : 1) un sens de soi incarné et positif par la construction identitaire et le dépassement des séquelles de la transphobie; 2) la confiance en soi par des liens interpersonnels validants; 3) l'estime de soi par la fierté d'être trans et l'ancrage communautaire; 4) le respect de soi par la possibilité de vivre dans le genre désiré en sécurité et avec équité.
This document is protected by copyright law. Use of the services of Erudit (including reproduction) is subject to its terms and conditions, which can be viewed online.

https://apropos.erudit.org/en/users/policy-on-use/ 


\section{Un modèle pour les interventions transaffirmatives}

Les jeunes trans et non-binaires (JTNB) ne se sentent pas confortables dans le sexe/genre qui leur a été assigné à la naissance et ne s'y identifient pas (Richards, 2020). Cette non-identification au genre assigné entraîne des discriminations, oppressions structurelles ainsi qu'un stress minoritaire (Meyer, 2013) qui s'exercent tout au long de leur développement puisqu'iels grandissent dans une société hétérocisnormée construite sur une conception binaire du genre. Par la notion de sexe/genre, nous faisons référence à un système symbolique, social et politique qui donne aux individus un destin social en fonction du sexe qui leur est assigné (Butler, 1990). II prescrit ce qu'iels sont censés vivre, ressentir et exprimer, il définit les corps et distribue inéquitablement les pouvoirs et les ressources économiques. Si ce système est vécu comme suffisamment adéquat par certain.e.x.s - les personnes dites cisgenres, il est par contre inadapté pour les personnes trans, non binaires, agenres ou fluides (Medico, 2019; Richards et al., 2016). Ceci implique pour les JTNB de se construire comme individu en dehors des discours sociaux dominants et de devoir en porter les conséquences identitaires, sociales, relationnelles, corporelles, médicales, économiques, juridiques, politiques et symboliques (Medico, 2020). Pour accompagner ces jeunes vers un mieux-être et une affirmation positive de soi, des approches transaffirmatives sont actuellement en développement dans plusieurs champs de l'intervention sociale et de santé (Ehrensaft, 2011; Pullen Sansfaçon et Medico, 2021; Riggs, 2019; Spivey et Edwards-Leeper, 2019). Ces approches se développent en parallèle aux preuves scientifiques qui démontrent l'impact négatif sur la santé mentale des approches correctives ou «thérapies de conversion » (Ehrensaft et al., 2018; Leibowitz et al., 2020; Turban et Ehrensaft, 2018; Turban et al., 2020). Notre objectif est ici de participer à la fondation des bases théoriques et empiriques des approches transaffirmatives. Plus précisément nous proposons un modèle conceptuellement cohérent et basé sur les preuves concernant les facteurs de résilience chez les JTNB.

\section{Qui sont les jeunes trans et non binaires et quels sont leurs besoins}

Dans les pays ${ }^{1}$ où des études robustes ont évalué qui et combien de jeunes s'identifient comme transgenres, les proportions les plus récentes varient entre 1,2 et 2,7 \% selon la revue systématique et méta-analyse de Zhang et al. (2020). Toujours selon cette étude, si une définition plus large est utilisée dans les enquêtes, telle qu'une incongruence et une ambivalence face au genre, les prévalences vont de 2,5 à 8,4\% (Zhang et al., 2020). Parmi ces jeunes une proportion grandissante se décrit comme non binaire et de genre fluide (Motmans et al., 2019; Taylor et al., 2020) et le ratio de personnes assignées fille à la naissance dépasse depuis quelques années celui des personnes assignées homme (Steensma et al., 2018).

À I'heure actuelle, on a tendance à considérer que les jeunes non-binaires, agenres et de genre fluide font partie de la catégorie des jeunes trans et, dans ce texte, nous suivrons cette tendance puisque nous mettons l'accent sur les oppressions que partagent les JTNB dans nos systèmes sociaux hétérocisnormatifs. Toutefois, les jeunes non binaires, agenres et de genre fluide sont encore moins conformes aux expressions stéréotypées et socialement acceptées du genre. Ceci les rend encore plus vulnérables aux discriminations et aux micro-agressions, notamment de type mégenrage, l'accès aux soins d'affirmation de genre leur est plus difficile et les interventions médicales sont moins adaptées à leurs besoins (Brennan et al., 2017; Motmans et al., 2019; Rimes et al., 2019). II semble que chez les jeunes qui demandent des traitements médicaux de confirmation de genre, les jeunes non binaires, de plus en plus nombreuxes (Twist et de Graaf, 2019) vivent plus d'anxiété, de dépression et présentent une moins bonne estime de soi que les jeunes trans binaires (Thorne et al., 2019).

Les écrits sont consensuels sur la vulnérabilité exacerbée des JTNB face à la souffrance psychique: les symptomatologies les plus rapportées sont la dépression, l'anxiété, les idéations et conduites suicidaires, les troubles alimentaires et les conduites autodestructrices et automutilatoires (Arcelus et al., 2016; Bauer et al., 2013; Clark et al., 2014; Connolly et al., 2016; Diemer et al., 2015; Olson, Schrager et al., 2015; Puckett et al., 2019; Reisner et al., 2016; Rimes et al., 2019; Röder et al., 2018; Watson et al., 2017). L'association entre cette vulnérabilité accrue et les discriminations, les oppressions structurelles et le stress minoritaire est également largement documentée dans les écrits tant sur des échantillons d'adolescent.e.x.s que sur des jeunes adultes (Bauer et al., 2015; Bockting et al., 2013; Bouman, Davey et al.; 2016; Brennan et al., 2017; Chodzen et al., 2019; Ehrensaft et al., 2018; Gorman et al., 2020; Hatchel et al, 2019; Testa et al., 2012, 2017; Veale et al., 2017; White Hughto et al., 2015). Cette symptomatologie semble la plus aigüe au moment de la puberté, mais il semble que même chez les enfants la potentielle suicidalité inquiète les parents (MacMullin et al.;

' La recension systématique des écrits et méta-analyse de Zhang et al. (2020) a retenu 19 publications basées sur des études provenant d'échantillons des États-Unis (13 études), de la Hollande (2) et une seule pour Suède, Belgique, Taiwan et Nouvelle-Zélande. 
2020). Le suicide est d'ailleurs une des questions les plus débattues pour cette population à très haut risque (Grossman et al., 2016; McNeil et al., 2017; Testa et al., 2012, 2017).

Développer des interventions qui permettent de dépasser les séquelles des oppressions et qui comprennent la souffrance des JTNB de manière globale s'impose (McNeil et al., 2017; Puckett et al., 2019; Watson et Veale, 2018; Zeeman et al., 2017). L'objectif final est de donner un avenir à ces jeunes chez qui il a été documenté que les perspectives pour le futur, comme l'espoir d'avoir une place dans la société, un emploi ou une vie heureuse sont faibles, les JTNB se sentant aussi très incompris et rejetés par les adultes et les personnes cisgenres (Singh et al., 2014). C'est pourquoi les approches transaffirmatives visent essentiellement à favoriser la résilience. Le concept de résilience dans ce contexte correspond à ce que Singh (2013) définit dans une étude qualitative sur les mécanismes de résilience des jeunes trans racisés, soit : « [the] ability to «bounce back» from challenging expériences» (p. 692).

\section{Un modèle basé sur l'éthique de la reconnaissance et les preuves scientifiques sur la résilience chez les JTNB}

Nous nous inscrivons dans les travaux de Sauvé et Pullen Sansfaçon (2021) qui dans un chapitre sur les fondements juridiques et éthiques des interventions transaffirmatives défendent l'idée que la théorie de la reconnaissance d'Axel Honneth $(2000,2006)$ offre un socle conceptuel nécessaire et cohérent avec les buts de l'approche transaffirmative.

Les travaux en philosophie politique d'Axel Honneth $(2000,2006)$ visent le développement d'une société plus juste et d'une «vie bonne » (Butler, 2012) pour toustes. L'auteur défend l'idée que l'expérience que les individus font de la reconnaissance de leur existence par les autres, soit dans l'espace intersubjectif, est nécessaire pour l'atteinte d'une société juste et équitable. Cette reconnaissance permet aux personnes de se reconnaitre elles-mêmes comme dignes d'avoir une existence, des projets et une «vie bonne». Selon Honneth, la reconnaissance sociale s'exprime à trois niveaux : les relations primaires (amour, amitié), les relations juridiques (droits), la communauté de valeurs (solidarité) - «l'expérience de l'amour donne ainsi accès à la confiance en soi, l'expérience de la reconnaissance juridique au respect de soi, et l'expérience de la solidarité, enfin [à] l'estime de soi » (Honneth, 2000, p. 290). II se réfère aussi aux travaux sur les relations d'objet en psychologie de Donald Winnicott (1965) et ceux de Daniel Stern (1998) sur l'intersubjectivité pour comprendre comment les mécanismes relationnels fondent la relation à soi.

Bien qu'Honneth ait mis implicitement la reconnaissance subjective au centre de son modèle, il n'a toutefois pas formalisé ce que serait cette reconnaissance subjective - ou relation à soi - car tel n'était ni son but ni son champ d'application. Or, pour les besoins d'une application en psychothérapie, nous avons jugé nécessaire de l'ajouter explicitement dans un modèle qui comprendrait ainsi quatre niveaux. Ces niveaux définissent quatre types d'objectifs pour les interventions transaffirmatives. Le niveau de relation à soi est un ajout de notre part, les trois autres sont repris d'Honneth (2006, p. 222). Ces objectifs peuvent être atteints en travaillant sur plusieurs questions et avec certains outils en fonction de ce que montrent les écrits actuels sur la résilience chez les JTNB. Le Tableau 1 résume les niveaux de reconnaissance, les objectifs d'intervention et les principales pistes d'intervention selon les écrits scientifiques.

Pour étoffer ce modèle, nous présentons une synthèse des pistes identifiées dans les écrits en les organisant par niveau de reconnaissance ainsi que des suggestions d'applications concrètes pour les interventions. Cette synthèse se base sur une revue narrative des écrits portant sur les facteurs de résilience chez les JTNB incluant, tant des travaux quantitatifs que qualitatifs de 2011 à $2021^{2}$. Seules les publications discutant spécifiquement des JTNB, en anglais et en français, ont été retenues. Nous n'avons pas retenu les travaux portant sur des modèles d'évaluation, des échelles de mesure et les descriptions de procédures médicales. Notre corpus se compose de 89 articles empiriques publiés dans des revues avec arbitrage, 20 recension des écrits, articles de synthèse et éditoriaux dans des revues avec arbitrage, cinq ouvrages écrits ou dirigés par des clinicien.ex.s et chercheur.e.x.s d'expérience sur les approches transaffirmatives et le développement de l'identité trans, deux rapports de recherche et un chapitre issu d'un ouvrage collectif (autre que les cinq ouvrages mentionnés).

\footnotetext{
${ }^{2}$ De manière générale, on observe dans les écrits scientifiques un changement de paradigme autour de 2011 - raison pour laquelle nous avons choisi cette date pour débuter la revue des écrits. Ceci est patent lorsque l'on compare les deux revues systématiques des écrits (Moradi et al., 2016; Tankersley et al., 2021).
} 
Tableau 1. Niveaux de reconnaissance, objectifs d'intervention et principales pistes d'intervention pour favoriser la résilience des JTNB selon les écrits scientifiques.

\begin{tabular}{|c|c|c|}
\hline Niveaux de reconnaissance & Objectifs d'intervention & Principales pistes d'intervention \\
\hline $\begin{array}{l}\text { Subjective: } \\
\text { relation à soi }\end{array}$ & $\begin{array}{l}\text { Un sens de soi incarné et } \\
\text { positif }\end{array}$ & $\begin{array}{l}\text { Construire une identité de genre confortable } \\
\text { Développer un rapport positif au corps } \\
\text { Dépasser les impacts de la transphobie et de la transphobie intériorisée }\end{array}$ \\
\hline $\begin{array}{l}\text { Intersubjective : } \\
\text { relations primaires }\end{array}$ & La confiance en soi & $\begin{array}{l}\text { Être reconnu.e.x dans son genre } \\
\text { Être soutenu.e.x par sa famille/parents } \\
\text { Être soutenu.ex par ses ami.e.x.s et se sentir connecté.e.x à son école } \\
\text { Pouvoir développer une intimité affective et sexuelle positive }\end{array}$ \\
\hline $\begin{array}{l}\text { Communautaire : } \\
\text { communauté de valeurs }\end{array}$ & $\begin{array}{l}\text { L'estime de soi par la } \\
\text { fierté d'être trans }\end{array}$ & $\begin{array}{l}\text { S'engager dans des groupes de JTNB } \\
\text { Trouver un espace sécuritaire pour s'exprimer et partager (groupe } \\
\text { communautaire ou réseaux sociaux) } \\
\text { Pouvoir accéder à des modèles positifs }\end{array}$ \\
\hline $\begin{array}{l}\text { Légale: } \\
\text { relations juridiques }\end{array}$ & Le respect de soi & $\begin{array}{l}\text { Avoir la possibilité de vivre dans son genre désiré en sécurité et en étant } \\
\text { reconnu socialement et légalement dans tous les contextes de vie } \\
\text { Avoir accès aux soins d'affirmation de genre avec des professionnel.I.e.x.S } \\
\text { compétent.e.X.s }\end{array}$ \\
\hline
\end{tabular}

\section{La reconnaissance subjective}

L'objectif de ce niveau d'intervention est de permettre aux jeunes de construire un sens de soi positif. Le sens de soi est un concept de psychologie humaniste provenant de Carl Rogers (1959) qui recoupe l'estime de soi, les projections de soi et l'image de soi. II s'agit d'éléments fondamentaux pour les bases d'un bon fonctionnement psychique et relationnel. Dans cette optique, la construction de soi et de son identité, le fait que celle-ci soit incarnée, donc vécue corporellement, mais aussi émotionnellement, fait en sorte que dans les facteurs subjectifs que l'on retrouve dans les travaux abordent principalement trois questions: 1) la construction d'une identité de genre confortable; 2) le rapport au corps et le sentiment positif envers soi; 3 ) le dépassement des impacts de la transphobie et de la transphobie intériorisée.

La construction d'une identité de genre confortable est probablement l'enjeu premier des JTNB. L'on sait que le développement d'un sentiment d'identité de genre différent de celui assigné à la naissance peut parfois apparaître dès l'âge de 3-4 ans (Ehrensaft, 2011) et en moyenne survient entre les âges de 5 et 8 ans (Beemyn et Rankin, 2011; Olson, Schrager et al., 2015). Les travaux qualitatifs sur les JTNB indiquent que la possibilité de s'identifier comme personne non cisgenre est le premier pas vers un mieux-être et que cela est un processus (Budge et al., 2018; Kuper et al., 2018; Medico, Pullen Sansfaçon et al., 2020; Singh, 2013). Les jeunes racontent le développement de leur identité en identifiant le plus souvent comme origine un profond malaise, une souffrance, un sentiment de ne pas correspondre à ce qui est attendu et de ne pas se sentir comme les autres. Ensuite iels parlent d'un cheminement intérieur pour se comprendre, mettre des mots, se reconnaitre et pour avoir le courage de le dire. Ce cheminement jusqu'au coming out peut prendre plusieurs années, mais est le plus souvent vécu en secret.

Dans l'une des premières études quantitatives sur un échantillon représentatif mené en Nouvelle-Zélande auprès de 8166 jeunes âgés de 13 à 18 ans, 96 ont dit être transgenres, parmi eux 27,3 \% l'ont su avant l'âge de 8 ans, $17,9 \%$ entre 8 et 11 ans et 54,8 \% à 12 ans ou plus (Clark et al., 2014). Dans cette même étude, 65,2\% ne l'avaient dit à personne avant de répondre au questionnaire. Se concentrer sur le moment de la révélation à l'entourage n'est pas suffisant pour comprendre les parcours de vie puisqu'une partie importante des jeunes cache leurs questionnements, ou même la certitude d'être trans, pendant des années. Dès l'âge préscolaire, les enfants comprennent les stéréotypes de genre et la pression de s'y conformer (Carver et al., 2003) et tous les enfants qui se sentent différent.e.x.s dans le genre ne le ressentent ni de la même façon, ni n'ont la même envie ou possibilité de l'exprimer. Beaucoup, surtout lorsque ce sentiment devient clair entre 6 et 11 ans, auront peur d'être rejeté.e.x.s ou menacé.e.x.s s'iels en parlent ou le montrent par leurs comportements et leurs choix vestimentaires (Riley et al., 2011, 2013). Ceci remet en question la division classiquement proposée dans les milieux cliniques pour juger de qui peut avoir accès à des démarches médicales d'affirmation du genre en créant deux sous-groupes de JNTB : celleux qui rapportent une dysphorie de genre tôt dans l'enfance (early onset) et celleux qui la rapportent à partir de la puberté (late onset) (Cohen-Kettenis et Klink, 2015; Steensma et al., 2011, 2013). 
jeunes et leurs parents dans deux cohortes en Suisse et au Canada (Medico, Pullen Sansfaçon et al., 2020; Pullen Sansfaçon et al., 2020). Ceci a permis d'identifier au moins trois parcours développementaux distincts. Le premier, et le moins fréquent, est celui des enfants qui ont affirmé très tôt leur différence de genre, soit à l'âge préscolaire. lels l'ont fait avant de comprendre le poids des normes de genre et dans des familles où leur expression de genre créative était possible. Le deuxième parcours est celui de jeunes qui ont passé leur enfance dans des conflits internes et dans le secret, en questionnement ou en espérant que leur sentiment d'incongruence de genre disparaisse. Ce groupe a rapporté avoir toujours su que quelque chose n'allait pas, qu'iels n'étaient pas cisgenres (" comme les autres »), mais cela était difficile à comprendre ou trop dur à révéler. lels rapportent une profonde souffrance qui n'a commencé à s'apaiser que lorsqu'iels ont pu mettre des mots et exprimer le fait qu'iels n'étaient pas cisgenres. Finalement, un troisième parcours est celui des jeunes qui n'avaient pas vécu de questionnements pendant l'enfance, soit parce qu'iels ne se sentaient pas concernés par la question du genre, soit parce qu'iels pouvaient exprimer leur genre sans réticences, comme c'est souvent le cas des enfants assignés filles qui adoptent des comportements que l'entourage qualifie de masculins. Dans ce parcours, c'est l'arrivée de la puberté qui les a porté.e.x.s à questionner leur genre, puis à affirmer leur différence. En conclusion il n'existe pas de parcours identitaire unique, mais ce parcours est souvent associé à de la souffrance, à de profonds questionnements, à une expérience de devoir cacher qui l'on se sent être et au besoin de trouver une identité confortable.

Une autre des principales caractéristiques des JTNB est de vivre un profond et douloureux malaise par rapport aux caractéristiques sexuelles primaires et secondaires du corps (Röder et al., 2018). Ceci est particulièrement saillant au moment de la puberté et est associé aux difficultés psychiques et identitaires (Bouman, Davey et al., 2016). Cette « dysphorie corporelle » est un obstacle majeur à la découverte de la sexualité (Steensma et al., 2011), mais aussi une souffrance et un obstacle dans leur vie courante, les obligeant à porter par exemple des vestes de compression (binders) et à cacher leur corps. Elle est l'une des principales motivations à entreprendre des modifications corporelles hormonales ou chirurgicales (Bouman, de Vries et T'Sjoen, 2016). Ces traitements améliorent significativement l'image corporelle et le bien-être psychologique (Budge et al., 2013; Durwood et al., 2017; Olson et al., 2011; Olson, Key et al., 2015; Owen-Smith et al., 2018; Pullen Sansfaçon, Medico, Riggs et al., sous presse). Toutefois, certains jeunes semblent avoir des barrières internes les empêchant d'avoir recours à ces traitements : Goldenberg et al. (2020) montrent que les JTNB ayant une forte anticipation de stigmatisation par les médecins recourent moins aux soins d'affirmation de genre.

La transphobie s'accompagne concrètement d'expériences vécues par les jeunes telles que des situations de rejets, de discriminations et de violence. Ceci a un impact sur l'apprentissage des relations interpersonnelles en nuisant au sentiment interne de sécurité et de confiance en soi et envers les autres (Johnson et al., 2014; Mitchell et al., 2014). Lindley et Paz Galupo (2020) proposent que les pensées et émotions engendrées par des expériences directes et indirectes de discriminations, comme l'anticipation de situations de stigmatisation, la transphobie intériorisée ou le fait de devoir constamment cacher son identité de genre authentique, se répercutent sur une faible estime de soi, une perception négative de soi et des comportements autodestructeurs comme des automutilations, des conduites à risque et tentatives de suicide (Arcelus et al., 2016; Chodzen et al., 2019; Durwood et al., 2017; Scandurra et al., 2020).

La transphobie intériorisée semble aussi avoir un impact sur la manière de gérer les émotions (Budge et al., 2021; Grossman et al., 2011). Lindley et Paz Galupo (2020) ont montré que les JTNB ont des émotions plus fortes lorsqu'iels sont confronté.e.x.s à des émotions négatives que positives et qu'iels ont plus de difficultés à décrire les émotions positives. Cette gestion des émotions entraîne des difficultés personnelles et relationnelles.

\section{Interventions transaffirmatives au niveau subjectif}

À ce niveau nous visons essentiellement des interventions de type psychothérapie et accompagnement psychosocial. Ceux-ci doivent déconstruire l'impact des oppressions, soit la transphobie, mais aussi la transphobie intériorisée. Les discriminations concrètes, mais aussi indirectes, comme ne pas avoir la possibilité de comprendre son propre malaise, vivre dans le secret et devoir cacher qui l'on se sent être nuisent au développement d'un sens de soi positif (Medico, Galantino et al., 2020). Pour les JTNB garder leur identité de genre secrète leur permet d'éviter le rejet et la victimisation, mais entraîne des conséquences pour le développement des liens de confiance et de l'estime de soi. Un accompagnement transaffirmatif vise l'exploration des différentes possibilités de vivre le genre afin que les JTNB puissent expérimenter ce qui leur convient et dépasser leur propre transphobie intériorisée (Ehrensaft, 2011; Susset et Rabiau, 2021a, 2021b).

Avant la puberté, la meilleure attitude thérapeutique est de permettre à l'enfant d'explorer son genre en sécurité et il n'y a pas d'interventions spécifiques ni médicales ni psychologiques à mettre en oeuvre. Ces enfants ont 
surtout besoin d'un contexte de sécurité et de confiance dans leur famille, à l'école et dans tous les contextes de vie (Susset et Rabiau, 2021a). Le travail clinique consiste surtout à communiquer de l'information basée sur les preuves scientifiques et les standards de soin (voir WPATH), faire comprendre l'importance de genrer l'enfant en fonction de ses demandes, soutenir les familles et dépister des situations à risque chez les enfants (anxiété, traumas et violences, suicide) ou dans la famille (maltraitance, dissensions familiales sévères sur l'attitude à adopter, détresse des parents ou de la fratrie). La décision pour leur genre s'imposera de soi, le plus souvent à la puberté, et, pour ces enfants, savoir que des possibilités médicales existent est un grand soulagement.

À la puberté, une partie des JTNB prendra une décision ou fera un coming out avec soulagement et fierté : certains parlent d'une euphorie de genre. D'autres seront dans de profonds questionnements et d'autres encore manifesteront un état de crise avec une symptomatologie complexe et souvent sévère que l'on diagnostique sous l'appellation de « dysphorie de genre » (appellation du DSM-5 selon I'American Psychiatric Association, 2013) ou " incongruence du genre » (appellation de la CIM-11 selon I'Organisation Mondiale de la Santé, 2020). Cette souffrance cliniquement significative s'accompagne souvent d'une symptomatologie telle que menaces et/ou tentatives suicidaires, troubles alimentaires, automutilations, dépression, anxiété, rapport très difficile aux parties genrées de son corps. D'autres encore seront dans un profond questionnement identitaire avec des enjeux relationnels et communautaires importants. Dans tous les cas, la prise en compte rapide des besoins et une compréhension développementale sera nécessaire, car le moment le plus dangereux en termes de suicidalité est celui entre la première demande d'aide et le moment où cette aide arrive (Bauer et al., 2013). Les traitements médicaux d'affirmation de genre avec des bloqueurs de puberté peuvent être utilisés à partir du début de la puberté. Ces bloqueurs permettent de ralentir momentanément la puberté et préservent ainsi une possibilité de choix pour le futur, ce traitement est réversible (Ehrensaft et al., 2018; Gosh et Gorgos, 2021; Olson et al., 2011). L'introduction d'hormones (testostérone ou oestrogène) se fera plus tardivement en fonction des réalités somatiques et psychologiques des jeunes, car elle n'est que partiellement réversible. Les recherches menées depuis plus de 20 ans ont démontré l'efficacité et l'avantage coût/bénéfice sur le plan psychologique, social et somatique des traitements par bloqueurs et hormonaux (CohenKettenis et al., 2011; Pullen Sansfacon et al., 2018; Wiepjes et al., 2018). Des interventions chirurgicales comme une torsoplastie (dès 16 ans au Québec, variable dans les autres pays francophones) ou une augmentation mammaire et des interventions génitales sont également possibles dès la majorité légale dans la plupart des pays.

Lorsque les multiples oppressions entraînent une difficulté à mobiliser la résilience (p. ex., gestion des émotions, difficultés relationnelles, estime de soi défaillante, PTSD), un accompagnement psychothérapeutique transaffirmatif est particulièrement pertinent. Bien évidemment celui-ci doit dépendre de l'adhésion de la personne au suivi et combine des techniques thérapeutiques usuelles pour ces problématiques. Pour favoriser la résilience, il a été suggéré que la prise de conscience de l'intériorisation des discriminations, soit la «mentalisation du stigma » (pour reprendre les termes utilisés dans certaines approches thérapeutiques) permette de diminuer les effets de cette transphobie intériorisée sur la santé mentale (Scandurra et al., 2020). Les thèmes de l'acceptation de soi, I'affirmation identitaire et du soutien sont à favoriser (Amodeo et al., 2018) ainsi qu'une perspective de travail en psychothérapie qui contextualise les difficultés, soit qui articule les dimensions sociales, relationnelles et individuelles (Matsuno et Israel, 2018). Lindley et Paz Galupo (2020) proposent des pistes d'intervention thérapeutique centrées autour de la gestion émotionnelle afin que les JTNB développent de meilleures habiletés à comprendre et gérer leurs émotions et à mieux identifier ce qui provient de l'internalisation des expériences négatives. Pour toustes les jeunes, une déconstruction des injonctions à correspondre aux normes sociales du genre est nécessaire pour que le parcours d'affirmation soit adapté à la pluralité des genres. Ceci vaut également pour les intervenant.e.x.s qui doivent faire un travail réflexif sur leurs propres représentations du genre si iels souhaitent travailler avec des JTNB afin de ne pas imposer volontairement ou involontairement leur vision du genre.

\section{La reconnaissance intersubjective}

L'intervention doit favoriser la possibilité pour les jeunes d'expérimenter des liens interpersonnels validants. Dans les écrits scientifiques, ceci a été principalement étudié par rapport au soutien familial et à la qualité des liens perçus avec l'entourage. Ces liens semblent des facteurs fondamentaux pour le bien-être psychologique (Arcelus et al., 2016; Bockting et al., 2013; Lenning et Buist, 2013; Röder et al., 2018; Stewart et al., 2018), mais aussi des facteurs de protection face aux discriminations et à leur intériorisation (Veale et al., 2017). Inversement, l'isolement émotionnel et social est une source de stress et de détresse psychologiques (de Vries et al., 2016). Selon une étude comparant des jeunes cisgenres ( $N=2060)$, trans ( $N=349)$ ou ayant d'autres identités de genre $(N=681)$, les jeunes non cisgenres 
rapportent moins de soutien familial perçu, moins d'affiliations religieuses et ceux qui rapportent moins de soutien social rapportent aussi plus de détresse psychologique (Lefevor et al., 2019).

Le soutien familial est l'aspect le plus étudié, car il est associé au bien-être, au fonctionnement cognitif, au sentiment identitaire et à la résilience des jeunes trans (Olson et al., 2016; Puckett et al., 2019; Pullen Sansfacon et al., sous presse; Riggs et al., 2019; Travers et al., 2012). Il est aussi associé positivement à la probabilité de vivre dans son genre (Weinhardt et al., 2019). Plus précisément le soutien parental, tel que perçu par les jeunes, se manifeste sous la forme d'expression d'amour, de conseils, de soins, mais aussi comme le soutien émotionnel lors du coming out et l'adoption du prénom et du pronom choisi (Andrzejewski et al., 2021). Mais le coming out trans n'est pas toujours simple pour les familles, et les parents rencontrent parfois de profondes ambivalences et anxiétés face à des décisions à prendre comme celle d'autoriser son enfant à exprimer son identité de genre choisie à l'extérieur ou permettre à son adolescent.e.x de commencer un traitement médical d'affirmation du genre (Pullen Sansfaçon, Medico, Riggs, et al., sous presse). De plus, même lorsque les familles se montrent acceptantes et soutenantes à l'intérieur de la sphère familiale, elles rencontrent des critiques et des jugements provenant de l'extérieur (famille élargie, voisinage, école, ami.e.x.s) (Medico, Galantino et al., 2020; Pullen Sansfaçon et al., 2015). Ainsi, les relations intrafamiliales ne dépendent pas uniquement des dynamiques familiales, mais aussi de cinq facteurs contextuels que sont l'école, la communauté dans laquelle vit la famille, le travail des parents, la religion et la famille élargie (Bhattacharya et al., 2020). De plus les parents et les jeunes doivent trouver des ressources adéquates comme des centres spécialisés, des thérapeutes ou des groupes de soutien, et dans beaucoup de cas l'accès à ces ressources est difficile voire compromis pour des raisons aussi triviales que l'inexistence de ressources appropriées, l'éloignement géographique ou le coût financier (Pullen Sansfaçon et al., 2015; Riley et al., 2011).

À l'adolescence, les jeunes sont aussi davantage tournés vers l'extérieur de la famille. Le soutien des ami.e.x.s et de la communauté trans (Puckett et al., 2019) ainsi que le sentiment de connexion avec son école (Veale et al., 2017) ont aussi été étudiés et ils semblent déterminants pour le bien-être des JTNB. Plus précisément, Weinhardt et al. (2019) montrent que le soutien des ami.e.x.s est associé à un meilleur sens de connexion aux autres et au fait de trouver que la vie à un sens.

L'entrée dans la vie sexuelle et amoureuse participe à la construction de soi et à la possibilité d'une reconnaissance intersubjective chez toustes les adolescent.e.x.s mais elle semble être souvent différemment vécue par les JTNB. Doorduin et Van Berlo (2014) soulignent que de nombreuses personnes trans n'ont pas pu vivre les étapes essentielles de leur développement sexuel et relationnel. Les hypothèses de compréhension sont multiples. D'abord, des séquelles traumatiques d'expériences de violence et d'abus sexuels pendant l'enfance et l'adolescence plus élevées chez les JTNB que dans la population générale (Clark et al., 2014; Mitchell et al., 2014; Rimes et al., 2019). Ensuite, une dysphorie corporelle particulièrement marquée qui nuit, tant à la relation à soi qu'à l'autre, et donc complexifie l'entrée dans la sexualité (Bouman, Davey et al., 2016; Girard et al., 2021). Les JTNB rencontrent des défis particuliers dans leur vie sexuelle et intime : iels doivent dépasser l'oppression intériorisée et s'affranchir de la reproduction des normes de genre usuelles de la sexualité hétérosexuelle cisgenre et le manque de modèles pour elleux et avec leurs partenaires (Bauer et al., 2018; Fox Tree-McGrath et al., 2018; Katz-Wise et al., 2016; lantaffi et Bockting, 2011; Stephenson et al., 2017). L'influence néfaste de stéréotypes dévalorisants les associant à des objets sexuels bizarres a aussi été rapportée (Tompkins, 2014). Parallèlement, la sexualité et les relations amoureuses sont aussi décrites par les travaux qualitatifs comme associés au bien-être des personnes concernées, comme des voies de résilience, d'affirmation et de renforcement identitaire (Fox Tree-McGrath et al., 2018; Moolchaem et al., 2015).

\section{Interventions transaffirmatives au niveau intersubjectif}

Les interventions doivent favoriser la reconnaissance dans les milieux de vie et encourager les JTNB à développer des moyens de socialisation. Ceci impliquera parfois un travail avec les familles qui ont aussi besoin d'être soutenues pour épauler leurs jeunes et qui rencontrent de nombreux obstacles. L'acceptation et le soutien par les familles sont souvent le résultat d'un cheminement qui peut être difficile, prendre du temps, commencer par un sentiment de choc et peut entraîner des conflits au sein de la sphère familiale et/ou entre les parents (Frappier, 2011; Pullen Sansfaçon et al., 2015; Pullen Sansfaçon, Medico, Gelly et al., sous presse). Des interventions en thérapie familiale (Susset et Rabiau, 2021a, 2021b), la référence à des groupes et communautés de pairs, de même que des groupes de thérapie ou de soutien sont ainsi à favoriser, tant pour les jeunes que pour leurs parents (Zufferey et Pullen Sansfaçon, 2021). II sera important de porter attention au fait que les JTNB soient reconnu.e.X.s dans le prénom et les pronoms qu'iels utilisent. Un counselling sexologique et de santé sexuelle pourrait s'avérer pertinent pour les accompagner vers la possibilité d'une vie sexuelle et amoureuse en dehors des stéréotypes et les aider à mieux gérer leur dysphorie 
corporelle et les séquelles de la transphobie (Girard et al., 2021).

\section{La reconnaissance communautaire}

Ce niveau de reconnaissance passe par le développement d'un sentiment de fierté d'être trans et/ou nonbinaire, un ancrage communautaire et la solidarité que ce soit dans des groupes présents dans leur région ou via les réseaux sociaux. Participer à des groupes de pairs, avoir des ami.e.x.s qui partagent l'expérience de transitude, trouver une place dans la communauté queer ou LGBTQ2S+ sont des facteurs de résilience (Barr et al., 2016; Faddoul et Baril, 2021; Singh, 2013; Singh et al., 2014). Dans ces espaces communautaires et sur internet, on parle de "safe space », soit d'endroits où les jeunes trouvent un sentiment d'espoir, d'appartenance, un lieu où échapper à la violence et au stigma ainsi que la possibilité de redonner aux autres (Austin et al., 2020). Par la vie communautaire, les JTNB expérimentent des liens positifs, mais apprennent également comment éviter des situations de transphobie, ce qui semble permettre de mieux gérer les stress liés au genre (Gorman et al., 2020). L'engagement dans une lutte pour les droits des personnes trans semble aussi favoriser l'estime de soi (Pullen Sansfaçon et al., 2018; Singh, 2013). Il faut préciser que l'engagement communautaire n'est pas toujours associé à un mieux-être (Breslow et al., 2015), probablement en raison des relations complexes et parfois conflictuelles au sein de certains groupes et de l'existence de narratifs dominants sur ce que devrait être l'identité trans (Bradford et Syed, 2019) qui excluent certains jeunes qui n'y correspondraient pas.

L'accès à des modèles d'identification positifs est nécessaire (Levitt et Ippolito, 2014; McConnell et al., 2016). Mais pour les jeunes qui ne parlent pas l'anglais, les modèles positifs dans les médias sociaux et traditionnels sont rares (Bosom et Medico, 2020). Les médias traditionnels francophones, du moins en Europe, diffusent des représentations encore trop souvent stéréotypées ou négatives (Espineira, 2021).

\section{Interventions transaffirmatives au niveau communautaire}

Les interventions doivent encourager les JTNB à participer à la vie communautaire et favoriser l'intervention par les pairs. Différents modes, comme la thérapie par les arts, sont utilisés (Abdellahi et Thibeault, 2021).

\section{La reconnaissance légale}

Ce niveau d'intervention vise à garantir aux JTNB la possibilité de vivre dans leur genre désiré en sécurité ainsi qu'à améliorer l'accès aux soins de santé généraux et de confirmation de genre (Torres et al., 2015). Cette reconnaissance légale s'améliore, mais de manière inégale et les JTNB sont encore victimes de harcèlement et de violence et ne se sentent pas toujours en sécurité à l'école (Hatchel et al., 2019; Raymond et al., 2015). Si les personnes trans sont plus à risque de vivre des traumas interpersonnels violents (agressions physiques, violences sexuelles) que les personnes cisgenres, ceci est d'autant plus fréquent chez les jeunes et les femmes trans racisées (Budge et al., 2016; Singh, 2013). Les travaux sur l'impact des discriminations et de la violence sur les JTNB proposent de parler de « victimisation » qui se mesure par l'intensité et la fréquence des violences verbales et physiques rapportées par une personne et qu'elle identifie comme étant liées à son genre (Ybarra et al., 2015). La victimisation à l'adolescence est identifiée dans les écrits comme ayant un lien avec des symptômes de stress post-traumatique, dépression, anxiété, idéations suicidaires et les échecs scolaires, ainsi que des répercussions au début de l'âge adulte sur le développement professionnel, la capacité à construire des liens interpersonnels positifs et le suicide (Breslow et al., 2015; McNeil et al., 2017; Timmins et al., 2017; White Hughto et al., 2015; Ybarra et al., 2015).

La situation varie selon les contextes de vie et donc en fonction des facteurs intersectionnels d'oppression (race, classe, genre, capacitisme) ainsi que du contexte juridique et moral des différentes aires géographiques. Il semble, selon une récente étude comparative incluant quatre pays (Angleterre, Australie, Canada, Suisse), que la souffrance psychologique rapportée soit moins importante dans l'échantillon canadien : les auteur.e.x.s font l'hypothèse que le meilleur accès aux soins et la reconnaissance sociale et légale des personnes trans puisse expliquer cette situation (Pullen Sansfaçon, Medico, Riggs et al., sous presse).

Les jeunes trans ont parfois besoin d'avoir accès à des soins d'affirmation de genre pour traiter leur dysphorie/incongruence de genre (Coleman et al., 2012; Ehrensaft et al., 2018; The Lancet, 2018). Or, l'accès aux soins de santé généraux, comme d'affirmation de genre, n'est pas facile, peut être inéquitable et n'est pas partout le même (Gridley et al., 2016; Rider et al., 2018). Les professionnel.le.x.s de la santé manquent souvent de formation sur les questions trans et parfois mégenrent intentionnellement ou involontairement les JTNB (Medico, Galantino et al., 2020; Pullen Sansfaçon, Medico, Riggs et al., sous presse; Singh et al., 2014). Ceci crée un climat de méfiance chez les 
personnes trans, notamment lorsqu'on observe que $66,8 \%$ des JTNB ne révèlent pas leur genre aux professionnel.le.x.s de la santé selon l'étude américaine LGBTQ National Teen Survey (McKay et Watson, 2019). Selon cette étude portant sur 5637 jeunes de 13-17 ans, ceci est encore plus marqué chez les jeunes non binaires et cet indicateur est associé à plus de difficultés psychologiques. Pour les jeunes racisés, l'expérience de stigmatisation dans le système de soins influence aussi négativement leur possibilité d'y avoir accès (Goldenberg et al., 2021).

\section{Interventions transaffirmatives au niveau légal}

Ainsi, pour favoriser la résilience chez les JTNB, il faut pouvoir adapter le contexte structurel, légal, social, scolaire et sanitaire afin de leur garantir la possibilité de vivre dans le genre désiré en sécurité. Ceci implique au moins une protection légale et une équité dans l'accès à l'école, au monde professionnel, aux soins et une protection visant leur sécurité dans l'espace social, quelle que soit l'origine sociale, ethnique ou la forme de genre (binaire, non binaire, fluide, neutre, autre). Les récentes avancées législatives au Québec, comme la possibilité de changer de nom et de genre, et la protection du genre dans la Charte des droits et liberté sont à souligner. L'accès aux soins généraux de santé peut aussi être amélioré en supprimant les obstacles administratifs basés sur une catégorisation par le genre et le manque de formation des intervenant.e.x.s. La sécurité dans les écoles et dans la vie quotidienne implique de lutter activement et explicitement contre la transphobie et les discriminations systémiques. Certes, ce niveau dépasse I'intervention clinique, mais ces questions sont cruciales, car les difficultés que les JTNB rencontrent sont en partie créées et maintenues par des fonctionnements institutionnels et sociaux oppressifs.

\section{Limites}

La recherche sur les JTNB est récente et de nombreux angles morts subsistent. Nous en savons encore très peu, voire rien, sur celleux qui sont les moins visibles, comme les enfants dont les familles répriment toute expression de genre qui ne serait pas conforme au genre assigné, ou sur les jeunes qui ne peuvent pas ou ne veulent pas s'identifier comme JTNB, qu'elles que soient leurs raisons (p. ex., des contextes de vie où une expression trans ou non binaire constitue un danger pour leur sécurité voire leur survie, des jeunes qui sont encore en processus de prise de conscience de leur genre). Nous savons seulement que les adolescent.e.x.s qui vivent dans des familles transphobes se retrouvent dans certains cas pris.e.x.s en charge par la DPJ ou sont en situation d'itinérance et que leur genre trans et non binaire entraine dans ces contextes un cumul de difficultés, notamment dans l'accès aux ressources (Robichaud et al., 2021; Shelton et al., 2018). Nous avons aussi encore très peu de recul sur la question des jeunes non binaires, agenres et de genre fluide puisqu'iels étaient pratiquement invisibles avant 2016, moment où l'on retrouve les premiers articles en faisant explicitement mention (voir Richards et al., 2016). Notons aussi que la non-binarité est un défi pour le suivi médical hormonal qui reste une manière binaire d'induire des modifications corporelles (Wahlen, 2021). Ceci relève d'une complexité éthique et médicale certaine en dehors des recommandations actuelles (Wahlen, 2021; Wren, 2019). Les prochains standards de soin de la WPATH, les Standards of Care 8, devraient combler ce manque.

Les contextes d'oppressions intersectionnelles commencent à être étudiés, mais peu de travaux sont encore publiés sur les jeunes racisé.e.x.s, en situation de handicap, migrant.e.x.s et provenant d'autres cultures qu'occidentales: la majeure partie des travaux proviennent en effet d'Amérique du Nord, d'Europe, Australie, Nouvelle-Zélande et quelques-uns d'Asie. La question de la classe sociale et du revenu des parents est aussi invisible dans les écrits scientifiques alors que l'accès aux soins est souvent une question financière.

Certaines pistes ont été plus étudiées et l'essentiel des efforts au niveau de la reconnaissance intersubjective a pour l'instant été mis sur les parents dans une vision très nucléaire de la famille, au sens de la présence d'un père et d'une mère. Nous savons également très peu sur la manière dont les fratries contribuent ou vivent la transitude. De même, les autres types de familles, les enjeux complexes dans des familles recomposées, homoparentales, monoparentales ou dans des structures sociales moins individualistes que la société occidentale, sont encore à étudier. 


\section{Conclusions : la reconnaissance à la base des interventions transaffirmatives}

Les JTNB rencontrent des oppressions tout au long de leur développement et envisager les difficultés de ces jeunes avec le concept d'oppression nous amène à réfléchir à comment les dépasser en fonction de leurs parcours et contextes de vie et, sur ce point, la théorie de la reconnaissance d'Axel Honneth $(2000,2006)$ offre un cadre épistémologiquement cohérent avec les théories de l'intersubjectivité et une vision située de l'identité. Ce modèle par niveau de reconnaissance nous permet d'intégrer les connaissances actuelles sur ce qui cause des souffrances et comment en sortir. II décrit des moyens pour mobiliser la résilience à plusieurs niveaux : subjectif, intersubjectif, communautaire et juridique. II est en cela une base pour les approches transaffirmatives.

Ces approches sont d'abord une posture éthique qui vise une société plus juste. Elles se basent sur une compréhension des conditions de vie menant au bien-être ou à la souffrance dans les rapports intersubjectifs et cela dans une perspective développementale. Elles peuvent donc être concrètement mises en œuvre sous diverses formes et être adaptées aux approches et outils que les clinicien.ne.x.s et les intervenant.e.x.s utilisent déjà dans leurs pratiques en santé mentale. Ces approches visent à soutenir les jeunes en prenant en compte leur contexte de vie et la diversité de leurs parcours et besoins (Pullen Sansfaçon et Medico, 2021; Spivey et Edwaards-Leeper, 2019). Ceci implique un accompagnement sur mesure pour explorer leur genre, renforcer leur estime de soi et leurs capacités à prendre une décision sur leur parcours d'affirmation et éventuellement le recours à des démarches médicales ou de changement d'identité, développer des capacités de gestion émotionnelle et relationnelle, traiter les éventuels traumas, donner un sens aux expériences de transphobie en les mentalisant et trouver une identité qui leur soit confortable et qui leur permette de développer une fierté d'être qui iels sont. Elles utilisent plusieurs types d'interventions et d'outils comme la psychothérapie individuelle et l'accompagnement médical, un accompagnement des familles et des thérapies de groupe, l'éducation dans les écoles et la construction de bases légales pour la sécurité et l'équité, la transformation des structures de soins pour garantir l'accès aux soins et la formation continue des professionnel.le.x.s de la santé. Elles nécessitent un travail réflexif chez les intervenant.e.x.s et la valorisation des ressources communautaires. Pour approfondir, les lecteurices pourront consulter l'ouvrage de Riggs et al. (2019) ainsi que celui en français de Pullen Sansfaçon et Medico (2021) qui ont regroupé l'expérience en approches transaffirmatives d'intervenant.e.x.s de multiples professions et dans une perspective alliant les fondements légaux et institutionnels, l'intervention sociale et les approches thérapeutiques médicales et psychologiques.

\section{Conflit d'intérêt}

Les auteurs ne dévoilent pas de conflits d'intérêt.

\section{Références}

\section{Bibliographie générale, introduction et recommandations cliniques}

American Psychiatric Association (2013). Diagnostic and statistical manual of mental disorders (5 ${ }^{\text {th }}$ ed.). https://doi.org/10.1176/appi.books.9780890425596

Butler, J. (1990). Gender trouble: Feminism and the subversion of identity. Routledge.

Butler, J. (2012). Qu'est-ce qu'une vie bonne? Payot.

Carver, P. R., Yunger, J. L., \& Perry, D. G. (2003). Gender identity and adjustment in middle childhood. Sex Roles, 49(3-4), 95-109. https://doi.org/10.1023/A:1024423012063

Cohen-Kettenis, P. T., \& Klink, D. (2015). Adolescents with gender dysphoria. Best Practice \& Research Clinical Endocrinology \& Metabolism, 29(3), 485-495. https://doi.org/10.1016/j.beem.2015.01.004

Cohen-Kettenis, P. T., Schagen, S. E., Steensma, T. D., de Vries, A. L., \& Delemarre-van de Waal, H. A. (2011). Puberty suppression in a gender-dysphoric adolescent: A 22-year follow-up. Archives of Sexual Behavior, 40(4), 843-847. https://doi.org/10.1007/s10508-011-9758-9

Coleman, E., Bockting, W., Botzer, M., Cohen-Kettenis, P., DeCuypere, G., Feldman, J., Fraser, L., Green, J., Knudson, G., Meyer, W. J., Monstrey, S., Adler, R. K., Brown, G. R., Devor, A. H., Ehrbar, R., Ettner, R., Eyler, E., Garofalo, R., Karasic, D. H., ... Zucker, K. (2012). Standards of care for the health of transsexual, transgender, and gender-nonconforming people, version 7 . International Journal of Transgenderism, 13(4), 165-232. https://doi.org/10.1080/15532739.2011.700873

Honneth, A. (2000). La lutte pour la reconnaissance. Folio Essais.

Honneth, A. (2006). La société du mépris. Vers une nouvelle théorie critique. La Découverte. 
Meyer, I. H. (2013). Prejudice, social stress, and mental health in lesbian, gay, and bisexual populations: Conceptual issues and research evidence. Psychology of Sexual Orientation and Gender Diversity, 1(S), 3-26. https://doi.org/10.1037/2329-0382.1.S.3

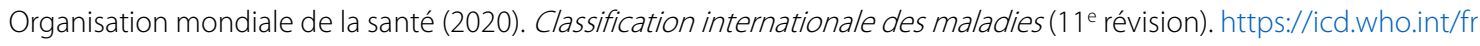

Rogers, C. (1959). A theory of therapy, personality and interpersonal relationships as developed in the client-centered framework. Dans S. Koch (dir.), Psychology: A study of a science. Formulations of the person and the social context (vol. 3, pp. 184-256). McGraw Hill.

Stern, D. (1998). The interpersonal world of the infant: A view from psychoanalysis and development psychology (2e ed.). Basic Books.

Thorne, N., Witcomb, G. L, Nieder, T., Nixon, E., Yip, A. \& Arcelus, J. (2019). A comparison of mental health symptomatology and levels of social support in young treatment seeking transgender individuals who identify as binary and non-binary. International Journal of Transgenderism, 20(2-3), 241-250. https://doi.org/10.1080/15532739.2018.1452660

Winnicott, D. W. (1965). The maturational processes and facilitating environment: Studies in the theory of emotional development. Karnac Books.

World Professional Association for Transgender Health (WPATH). (sous presse). Standards of Care version 8. https://www.wpath.org/soc8

Wren, B. (2019). Ethical issues arising in the provision of medical interventions for gender diverse children and adolescents. Clinical Child Psychology and Psychiatry, 24(2), 203-222. https://doi.org/10.1177/1359104518822694

Zhang, Q., Goodman, M., Adam, M., Corneil, T., Hashemi, L., Kreukels, B., Motmans, J., Snyder, R., \& Coleman, E. (2020). Epidemiological considerations in transgender health: A systematic review with focus on higher quality data. International Journal of Transgender Health, 21(2), 125-137. https://doi.org/10.1080/26895269.2020.1753136

\section{Bibliographie résilience - articles de recherche}

Amodeo, A. L., Picariello, S., Valerio, P., \& Scandurra, C. (2018). Empowering transgender youths: Promoting resilience through a group training program. Journal of Gay \& Lesbian Mental Health, 22(1), 3-19. https://doi.org/10.1080/19359705.2017.1361880

Andrzejewski, J., Pampati, S., Steiner, R. J., Boyce, L., \& Johns, M. M. (2021). Perspectives of transgender youth on parental support: Qualitative findings from resilience and transgender youth study. Health Education \& Behavior, 48(1), 74-81. https://doi.org/10.1177/1090198120965504

Austin, A., Craig, S. L., Navega, N., \& Mclnroy, L. B. (2020). It-s my safe space: The life-saving role of the internet in the lives of transgender and gender diverse youth. International Journal of Transgender Health, 21(1), 33-44. https://doi.org/10.1080/15532739.2019.1700202

Arcelus, J., Claes, L., Witcomb, G. L., Marshall, E., \& Bouman, W. P. (2016). Risk factors for non-suicidal self-injury among trans youth. Journal of Sexual Medicine, 13(3), 402-412. https://doi.org/10.1016/j.jsxm.2016.01.003

Barr, S. M., Budge, S. L., \& Adelson, J. L. (2016). Transgender community belongingness as a mediator between transgender selfcategorization and well-being. Journal of Counseling Psychology, 63(1), 87-97. https://doi.org/10.1037/cou0000127

Bauer, G., Pyne, J., Francino, M. C., \& Hammond, R. (2013). Suicidality among trans people in Ontario: Implications for social work and social justice. Service social, 59(1), 35-62. https://doi.org/10.7202/1017478ar

Bauer, G. R., Redman, N., Bradley, K., \& Scheim, A. I. (2018). Sexual health of trans men who are gay, bisexual, or who have sex with men: Results from Ontario, Canada. International Journal of Transgenderism, 14(2), 66-74. https://doi.org/10.1080/15532739.2013.791650

Bauer, G. R., Scheim, A. I., Pyne, J., Travers, R., \& Jammond, R. (2015). Intervenable factors associated with suicide risk in transgender persons: A respondent driven sampling study in Ontario, Canada. BMC Public Health, 15(1), Article 525. https://doi.org/10.1186/s12889-015-1867-2

Bhattacharya, N., Budge, S. L., Pantalone, D. W., \& Katz-Wise, S. L. (2020). Conceptualizing relationships among transgender and gender diverse youth and their caregivers. Journal of Family Psychology. 35(5), 595-605. https://doi.org/10.1037/fam0000815

Bockting, W. O., Miner, M. H., Swinburne Romine, R. E., Hamilton, A., \& Coleman, E. (2013). Stigma, mental health, and resilience in an online sample of the US transgender population. American Journal of Public Health, 103(5), 943-951. https://doi.org/10.2105/AJPH.2013.301241

Bosom, M., \& Medico, D. (2020). My first year on testosterone: Analyzing the trans experience through YouTube channels. Sexologies. 30(2), e83-e85. https://doi.org/10.1016/j.sexol.2020.10.001

Bouman, W. P., Davey, A., Meyer, C., Witcomb, G. L., \& Arcelus, J. (2016). Predictors of psychological well-being among trans individuals. Sexual and Relationship Therapy, 37(3), 359-375. https://doi.org/10.1080/14681994.2016.1184754

Bouman, W. P., de Vries, A. L., \& T'Sjoen, G. (2016). Gender dysphoria and gender incongruence: An evolving interdisciplinary field. International Review of Psychiatry, 28(1), 1-4. https://doi.org/10.3109/09540261.2016.1125740

Bradford, N. J., \& Syed, M. (2019). Transnormativity and transgender identity development: A master narrative approach. Sex Roles, 
81(5), 306-325. https://doi.org/10.1007/s11199-018-0992-7

Brennan, S. L., Irwin, J., Drincic, A., Amoura, N. J., Randall, A., \& Smith-Sallans, M. (2017). Relationship among gender-related stress, resilience factors, and mental health in a Midwestern U.S. transgender and gender-nonconforming population. International Journal of Transgenderism, 18(4), 433-445. https://doi.org/10.1080/15532739.2017.1365034

Breslow, A. S., Brewster, M. E., Velez, B. L., Wong, S., Geiger, E., \& Soderstrom, B. (2015). Resilience and collective action: Exploring buffers against minority stress for transgender individuals. Psychology of Sexual Orientation and Gender Diversity, 2(3), 253265. https://doi.org/10.1037/sgd0000117

Budge, S. L., Adelson, J. L., \& Howard, K. A. (2013). Anxiety and depression in transgender individual: The roles of transition status, loss, social support, and coping. Journal of Consulting and Clinical Psychology, 81(3), 545-557. https://doi.org/10.1037/a0031774

Budge, S. L., Belcourt, S., Conniff, J., Parks, R., Pantalone, D. W., \& Katz-Wise, S. L. (2018). A grounded theory study of the development of trans youths' awareness of coming with gender identity. Journal of Child and Family Studies, 27(9), $3048-3061$. https://doi.org/10.1007/s10826-018-1136-y

Budge, S. L., Orzechowski, M., Schams, A., Lavender, A., Onsgard, K., Leibowitz, S., \& Katz-Wise, S. L. (2021). Transgender and gender nonconforming youths' emotions: The appraisal, valence, arousal model. The Counselling Psychologist, 49(1), $138-172$. https://doi.org/10.1177/2F0011000020945051

Budge, S. L., Thai, J. L., Tebbe, E. A., \& Howard, K. A. (2016). The intersection of race, sexual orientation, socioeconomic status, trans identity, and mental health outcomes. The Counseling Psychologist, 44(7), $1025-1049$. https://doi.org/10.1177/0011000015609046

Chodzen, G., Hidalgo, M. A., Chen, D., \& Garofalo, R. (2019). Minority stress factors associated with depression and anxiety among transgender and gender non-conforming youth. Journal of Adolescent Health, 64(4), $467-471$. https://doi.org/10.1016/j.jadohealth.2018.07.006

Clark, T. C., Lucassen, M. F., Bullen, P., Denny, S. J., Fleming, T. M., Robinson, E. M., \& Rossen, F. V. (2014). The health and well-being of transgender high school students: Results from the New Zealand adolescent health survey (Youth'12). Journal of Adolescent Health, 55(1), 93-99. https://doi.org/10.1016/j.jadohealth.2013.11.008

Connolly, M. D., Zervos, M. J., Barone II, C. J., Johnson, C. C., \& Joseph, C. L. (2016). The mental health of transgender youth: Advances in understanding. Journal of Adolescent Health, 59(5), 489-495. https://doi.org/10.1016/j.jadohealth.2016.06.012

de Vries, A. L., Steensma, T. D., Cohen-Kettenis, P. T., VanderLaan, D. P., \& Zucker, K. J. (2016). Poor peer relations predict parent- and self-reported behavioral and emotional problems of adolescents with gender dysphoria: A cross-national, cross-clinic comparative analysis. European Child \& Adolescent Psychiatry, 25(6), 579-588. https://doi.org/10.1007/s00787-015-0764-7

Diemer, E. W., Grant, J. D., Munn-Chernoff, M. A., Patterson, D. A., \& Duncan A. E. (2015). Gender identity, sexual orientation, and eatingrelated pathology in a national sample of college students. Journal of Adolescent Health, 57(2), 144-149. https://doi.org/10.1016/j.jadohealth.2015.03.003

Doorduin, T., \&Van Berlo, W. (2014). Trans people's experience of sexuality in the Netherlands: a pilot study. Journal of Homosexuality, 61(5), 654-672. https://doi.org/10.1080/00918369.2014.865482

Durwood, L., McLaughlin, K. A., \& Olson, K. R. (2017). Mental health and self-worth in socially transitioned transgender youth. Journal of the American Academy of Child \& Adolescent Psychiatry, 56(2), 116-123.e2. https://doi.org/10.1016/j.jaac.2016.10.016

Fox Tree-McGrath, C. A., Puckett, J. A., Reisner, S. L., \& Pantalone, D. W. (2018). Sexuality and gender affirmation in transgender men who have sex with cisgender men. International Journal of Transgenderism, 19(4), $389-400$. https://doi.org/10.1080/15532739.2018.1463584

Goldenberg, T., Gamarel, K. E., Reisner, S. L., Jadwin-Cakmak, L., \& Harper, G. W. (2021). Gender affirmation as a source of resilience for addressing stigmatizing healthcare experiences of transgender youth of color. Annals of Behavioral Medicine. Advance online publication. https://doi.org/10.1093/abm/kaab011

Goldenberg, T., Kahle, E. M., \& Stephenson, R. (2020). Stigma, resilience, and health care use among transgender and other gender diverse youth. Transgender Health, 5(3), 173-181. https://doi.org/10.1089/trgh.2019.0074

Gorman, K. R., Shipherd, J. C., Collins, K. M., Gunn, H. A., Rubin, R. O., Rood, B. A., \& Pantalone, D. W. (2020). Coping, resilience, and social support among transgender and gender diverse individuals experiencing gender-related stress. Psychology of Sexual Orientation and Gender Diversity. Advance online publication. https://doi.org/10.1037/sgd0000455

Gosh, S., \& Gorgos, A. (2021). L'accompagnement pédiatrique et les traitements hormonaux. Dans A. Pullen Sansfaçon \& D. Medico (dir.), Jeunes trans et non binaires : de l'accompagnement à l'affirmation (pp. 323-339). Éditions Remue-Ménage.

Gridley, S. J., Crouch, J. M., Evans, Y., Eng, W., Antoon, E., Lyapustina, M., Schimmel-Bristow, A., Woodward, J., Dundon, K., Schaff, R., McCarty, C., Ahrens, K., \& Breland, D. J. (2016). Youth and caregiver perspectives on barriers to gender-affirming health care for transgender youth. Journal of Adolescent Health, 59(3), 254-261. https://doi.org/10.1016/j.jadohealth.2016.03.017

Grossman, A. H., D'Augelli, A. R., \& Frank, J. H. (2011). Aspects of psychological resilience among transgender youth. Journal of LGBT 
Youth, 8(2), 103-115. https://doi.org/10.1080/19361653.2011.541347

Grossman, A. H., Park, J. Y., \& Russell, S. T. (2016). Transgender youth and suicidal behaviors: Applying the interpersonal psychological theory of suicide. Journal of Gay \& Lesbian Mental Health, 2044), 329-349. https://doi.org/10.1080/19359705.2016.1207581

Hatchel, T., Valido, A., De Pedro, K. T., Huand, Y., \& Espelage, D. L. (2019). Minority stress among transgender adolescents: the role of peer victimization, school belonging, and ethnicity. Journal of Child and Family Studies, 28(9), $2467-2476$. https://doi.org/10.1007/s10826-018-1168-3

lantaffi, A., \& Bockting, W. O. (2011). Views from both sides of the bridge? Gender, sexual legitimacy and transgender people's experiences of relationships. Culture, Health \& Sexuality, 13(3), 355-370. https://doi.org/10.1080/13691058.2010.537770

Johnson, C. W., Singh, A. A., \& Gonzalez, M. (2014). «It's complicated » : Collective memories of transgender, queer, and questioning youth in high school. Journal of Homosexuality, 61(3), 419-434. https://doi.org/10.1080/00918369.2013.842436

Katz-Wise, S. L., Reisner, S. L., Hughto, J. W., \& Keo-Meier, C. L. (2016). Differences in sexual orientation diversity and sexual fluidity in attractions among gender minority adults in Massachusetts. The Journal of Sex Research, 53(1), 74-84. https://doi.org/10.1080/00224499.2014.1003028

Kuper, L. E., Wright, L., \& Mustanski, B. (2018). Gender identity development among transgender and gender nonconforming emerging adults: An intersectional approach. International Journal of Transgenderism, 19(4), 436-455. https://doi.org/10.1080/15532739.2018.1443869

Lefevor, T. G., Sprague, B. M., Boyd-Rogers, C. C., \& Smack, A. C. P. (2019). How well do various types of support buffer psychological distress among transgender and gender nonconforming students? International Journal of Transgenderism, 20(1), 39-48, https://doi.org/10.1080/15532739.2018.1452172

Lenning, E., \& Buist, C. L. (2013). Social, psychological and economic challenges faced by transgender individuals and their significant others: Gaining insight through personal narratives. Culture, Health \& Sexuality, 15(1), 44-57. https://doi.org/10.1080/13691058.2012.738431

Levitt, H. M., \& Ippolito, M. R. (2014). Being transgender: The experience of transgender identity development. Journal of Homosexuality, 61(12), 1727-1758. https://doi.org/10.1080/00918369.2014.951262

Lindley, L., \& Paz Galupo, M. (2020). Gender dysphoria and minority stress: Support for inclusion of gender dysphoria as a proximal stessor. Psychology of Sexual Orientation and Gender Diversity, 73), 265-275. https://doi.org/10.1037/sgd0000439

MacMullin, L. N., Aitken, M., Nabbijohn, A. N., \& VanderLaan, D. P. (2020). Self-harm and suicidality in gender-nonconforming: A canadian community-based parent-report study. Psychology of Sexual Orientation and Gender Diversity, 71), 76-90. https://doi.org/10.1037/sgd0000353

Matsuno, E., \& Israel, T. (2018). Psychological interventions promoting resilience among transgender individuals: Transgender resilience intervention model (TRIM). The Counseling Psychologist, 46(5), 632-655. https://doi.org/10.1177/0011000018787261

McConnell, E. A., Birkett, M., \& Mustanski, B. (2016). Families matter: Social support and mental health trajectories among lesbian, gay, bisexual, and transgender youth. Journal of Adolescent Health, 596), 674-680. https://doi.org/10.1016/j.jadohealth.2016.07.026

McKay, T. R., \& Watson, R. J. (2019). Gender expansive youth disclosure and mental health: Clinical implications of gender identity disclosure. Psychology of Sexual Orientation and Gender Diversity, 7(1), 66-75. https://doi.org/10.1037/sgd0000354

Medico, D., Galantino, G., Zufferey, A., \& Pullen Sansfaçon, A. (2020). « J'aimerais mourir. » Comprendre le désespoir chez les jeunes trans par le concept d'oppression développementale. Frontières, 31(2). https://doi.org/10.7202/1070338ar

Medico, D., Pullen Sansfaçon, A., Zufferez, A., Galantino, G., Bosom, M., \& Suerich-Gulick, F. (2020). Pathways to gender affirmation in trans youth: A qualitative and participative study with youth and their parents. Clinical Child Psychology and Psychiatry, 25(4), 1002-1014. https://doi.org/10.1177/1359104520938427

Mitchell, K. J., Ybarra, M. L., \& Korchmaros, J. D. (2014). Sexual harassment among adolescents of different sexual orientations and gender identities. Child Abuse \& Neglect, 38(2), 280-295. https://doi.org/10.1016/j.chiabu.2013.09.008

Olson, K. R., Durwood, L., DeMeules, M., \& McLaughlin, K. A. (2016). Mental health of transgender children who are supported in their identities. Pediatrics, 137(3), Article e20153223. https://doi.org/10.1542/peds.2015-3223

Olson, K. R., Key, A. C., \& Eaton, N. R. (2015). Gender cognition in transgender children. Psychological Science, 26(4), 467-474. https://doi.org/10.1177/0956797614568156

Olson, J., Schrager, S. M., Belzer, M., Simons, L. K., \& Clark, L. F. (2015). Baseline physiologic and psychosocial characteristics of transgender youth seeking care for gender dysphoria. Journal of Adolescent Health, 57(4), 374-380. https://doi.org/10.1016/j.jadohealth.2015.04.027

Owen-Smith, A. A., Gerth, J., Sineath, R. C., Barzilay, J., Becerra-Culqui, T. A., Getahun, D., Giammattei, S., Hunkeler, E., Lash, T. L., Millman, A., Nash, R., Quinn, V. P., Robinson, B., Roblin, D., Sanchez, T., Silverberg, M. J., Tangpricha, V., Valentine, C., Winter, S., .. Goodman, 
M. (2018). Association between gender confirmation treatments and perceived gender congruence, body image satisfaction, and mental health in a cohort of transgender individuals. Journal of Sexual Medicine, 15(4), 591-600. https://doi.org/10.1016/j.jsxm.2018.01.017

Puckett, J. A., Matsuno, E., Dyar, C., Mustanski, B., \& Newcom, M. E. (2019). Mental health and resilience in transgender individuals: What type of support makes a difference? Journal of Family Psychology, 33(8), 954-964. https://doi.org/10.1037/fam0000561

Pullen Sansfaçon, A., Hébert, W., Ou Jin Lee, E., Faddoul, M., Tourki, D., \& Bellot, C. (2018). Digging beneath the surface: Results from stage one of a qualitative analysis of factors influencing the well-being of trans youth in Quebec. International Journal of Transgenderism, 19(2), 184-202. https://doi.org/10.1080/15532739.2018.1446066

Pullen Sansfaçon, A., Medico, D., Gelly, M., Kirichenko, V., \& Suerick Gullick, F. (sous presse). Blossoming child, mourning parent: A journey of adaptation and learning of trans youth and their parents accessing gender affirming care. Journal of Child and Family Studies.

Pullen Sansfaçon, A., Medico, D., Riggs, D., Carlile, A., \& Suerich-Gulick, F. (sous presse). Growing up trans in Canada, Switzerland, England, and Australia: Access to and impacts of gender-affirming medical care. Journal of LGBT Youth.

Pullen Sansfacon, A., Medico, D., Suerich-Gullick, F., \& Temple Newhook, J. (2020). "I knew that I wasn't cis, I knew that, but I didn't know exactly": Gender identity development, expression and affirmation in youth who access gender affirming medical care. International Journal of Transgender Health, 21(3), 307-320. https://doi.org/10.1080/26895269.2020.1756551

Pullen Sansfaçon, A., Robichaud, M-J., \& Dumais-Michaud, A. A. (2015). The experience of parents who support their children's gender variance. Journal of LGBT Youth, 12(1), 39-63. https://doi.org/10.1080/19361653.2014.935555

Raymond, G., Blais, M., Bergeron, F. A., \& Hébert, M. (2015). Les expériences de victimisation, la santé mentale et le bien-être de jeunes trans au Québec. Santé mentale au Québec, 403), 77-92. https://doi.org/10.7202/1034912ar

Rider, G. N., McMorris, B. J., Gower, A. L., Coleman, E., \& Eisenberg, M. E. (2018). Health and care utilization of transgender and gender nonconforming youth: A population-based study. Pediatrics, 141(3), Article e20171683. https://doi.org/10.1542/peds.20171683

Riggs, D. W., Bartholomaeus, C., \& Sansfaçon, A. P. (2019). "If they didn't support me, I most likely wouldn't be here": Transgender young people and their parents negotiating medical treatment in Australia. International Journal of Transgenderism, 21(1), 315. https://doi.org/10.1080/15532739.2019.1692751

Riley, E. A., Clemenson, L., Sitharthan, G., \& Diamond, M. (2013). Surviving a gender-variant childhood: The views of transgender adults on the needs of gender-variant children and their parents. Journal of Sex \& Marital Therapy, 39(3), $241-263$. https://doi.org/10.1080/0092623X.2011.628439

Riley, E. A., Sitharthan, G., Clemson, L., \& Diamond, M. (2011). The needs of gender-variant children and their parents: A parent survey. International Journal of Sexual Health, 23(3), 181-195. https://doi.org/10.1080/19317611.2011.593932

Rimes, K. A., Goodship, N., Ussher, G., Baker, D., \& West, E. (2019). Non-binary and binary transgender youth: Comparison of mental health, self-harm, suicidality, substance use and victimization experiences. International Journal of Transgenderism, 20(2-3), 230-240. https://doi.org/10.1080/15532739.2017.1370627

Röder, M., Barkmann, C., Richter-Appelt, H., Schulte-Markwort, M., Ravens-Sieberer, U., \& Becker, I. (2018). Health-related quality of life in transgender adolescents: Associations with body image and emotional and behavioral problems. International Journal of Transgenderism, 19(1), 78-91. https://doi.org/10.1080/15532739.2018.1425649

Scandurra, C., Dolce, P., Vitelli, R., Esposito, G., Testa, R. J., Balsam, K. F., \& Bochicchio, V. (2020). Mentalizinf stigma: Reflective functioning as a protective factor against depression and anxiety in transgender and gender-nonconforming people. Journal of Clinical Psychology, 76(9), 1613-1630. https://doi.org/10.1002/jclp.22951

Singh, A. A. (2013). Transgender youth of color and resilience: Negotiating oppression and finding support. Sex Roles, 68(11), 690702. https://doi.org/10.1007/s11199-012-0149-z

Singh, A. A., Meng, S. E., \& Hansen, A. W. (2014). "I am my own gender": Resilience strategies on trans youth. Journal of Counseling \& Development, 92(2), 208-218. https://doi.org/10.1002/j.1556-6676.2014.00150.x

Shelton, J., Wagaman, M. A., Small, L., \& Abramovich, A. (2018). I'm more driven now: Resilience and resistance among transgender and gender expansive youth and young adults experiencing homelessness. International Journal of Transgenderism, 19(2), 144-157. https://doi.org/10.1080/15532739.2017.1374226

Steensma, T. D., Biemond, R., de Boer, F., \& Cohen-Kettenis, P. T. (2011). Desisting and persisting gender dysphoria after childhood: A qualitative follow-up study. Clinical Child Psychology and Psychiatry, 16(4), $499-516$. https://doi.org/10.1177/1359104510378303

Steensma, T. D., Cohen-Kettenis, P. T., \& Zucker, K. J. (2018). Evidence for a change in the sex ration of children referred for gender dysphoria: Data from the center of expertise on gender dysphoria in Amsterdam (1988-2016). Journal of Sex \& Marital Therapy, 44(7), 713-715. https://doi.org/10.1080/0092623X.2018.1437580 
Steensma, T. D., Kreukels, B. P., de Vries, A. L., \& Cohen-Kettenis, P. T. (2013). Gender identity development in adolescence. Hormones and Behavior, 64(2), 288-297. https://doi.org/10.1016/j.yhbeh.2013.02.020

Testa, R., Hendricks, M. L., Bradford, J., \& Bongar, B. (2012). The relationship between gender-based victimization and suicide attempts in transgender people. Professional Psychology: Research and Practice, 43(5), 468-475. https://doi.org/10.1037/a0029605

Testa, R., Michaels, M. S., Bills, W., Rogers, M. L, Balsam, K. F., \& Joiner, T. (2017). Suicidal ideation in transgender people: Gender minority stress and interpersonal theory factors. Journal of Abnormal Psychology, 126(1), 125-136. https://doi.org/10.1037/abn0000234

Timmins, L., Rimes, K. A., \& Rahman, Q. (2017). Minority stressors and psychological distress in transgender individuals. Psychology of Sexual Orientation and Gender Diversity, 4(3), 328-340. https://doi.org/doi/10.1037/sgd0000237

Tompkins, A. B. (2014). «There's no chasing involved»: Cis/trans relationships, "tranny chasers", and the future of a sex-positive trans politics. Journal of Homosexuality, 61(5), 766-780. https://doi.org/10.1080/00918369.2014.870448

Torres, C. G., Renfrew, M., Kenst, K., Tan-McGrory, A., Betancourt, J. R., \& López, L. (2015). Improving transgender health by building safe clinical environments that promote existing resilience: Results from a qualitative analysis of providers. BMC Pediatrics, 15(1), Article 187. https://doi.org/10.1186/s12887-015-0505-6

Turban, J. L., Beckwith, N., Reisner, S. L., \& Keuroghlian, A. S. (2020). Association between recalled exposure to gender identity conversion efforts and psychological distress and suicide attempts among transgender adults. JAMA PSychiatry, 77(1), 68-76. https://doi.org/10.1001/jamapsychiatry.2019.2285

Twist, J., \& de Graaf, N. M. (2019). Gender diversity and non-binary presentations in young people attending the United Kingdom's National Gender Identity Development Service. Clinical Child Psychology and Psychiatry, 24(2), 277-290. https://doi.org/10.1177/1359104518804311

Veale, J., Peter, T., Travers, R., \& Saewyc, E. M. (2017). Enacted stigma, mental health, and protective factors among transgender youth in Canada. Transgender Health, 2(1), 207-216. https://doi.org/10.1089/trgh.2017.0031

Watson, R. J., Veale, J. F., \& Saewyc, E. M. (2017). Disordered eating behaviors among transgender youth: Probability profiles from risk and protective factors. International Journal of Eating Disorders, 5055), 515-522. https://doi.org/10.1002/eat.22627

Weinhardt, V. L., Xie, H., Wesp, L. M., Murray, J. R., Apchemengich, I., Kioko, D., Weinhardt C. B., \& Cook-Daniels, L. (2019). The role of family, friend, and significant other support in well-being among transgender and non-binary youth. Journal of GLBT Family Studies, 15(4), 311-325. https://doi.org/10.1080/1550428X.2018.1522606

Wiepjes, C. M., Nota, N. M., de Blok, C. J. M., Klaver, M., de Vries, A. L. C., Wensing-Kruger, S. A., de Jongh, R. T., Bouman, M-B., Steensma, T. D., Cohen-Kettenis, P., Gooren, L. J. G., Kreukels, B. P. C., \& den Heijer, M. (2018). The Amsterdam Cohort of Gender Dysphoria Study (1972-2015): Trends in prevalence, treatment, and regrets. Journal of Sexual Medicine, 15(4), $582-590$. https://doi.org/10.1016/j.jsxm.2018.01.016

Zeeman, L., Aranda, K., Sherriff, N., \& Cocking, C. (2017). Promoting resilience and emotional well-being of transgender young people: Research at the intersections of gender and sexuality. Journal of Youth Studies, 20(3), 382-397, https://doi.org/10.1080/13676261.2016.1232481

\section{Bibliographie résilience - recension des écrits, articles de synthèse et éditoriaux}

Ehrensaft, E., Giammattei, S. V., Storck, K., Tishelman, A. C., \& Keo-Meier, C. (2018). Prepubertal social gender transitions: What we know; what we can learn-A view from a gender affirmative lens. International Journal of Transgenderism, 19(2), $251-268$. https://doi.org/10.1080/15532739.2017.1414649

Leibowitz, S., Green, J., Massey, R., Boleware, A. M., Ehrensaft, D., Francis, W., Keo-Meier, C., Aydin Olson-Kennedy, A., Pardo, S., Rider, G. N., Schelling, E., Segovia, A., Tangpricha, V., Anderson, E., T'Sjoen G., \& on behalf of the WPATH, USPATH, and EPATH Executive Committee and Board of Directors (2020). Statement in response to calls for banning evidence-based supportive health interventions for transgender and gender diverse youth. International Journal of Transgender Health, 21(1), 111-112. https://doi.org/10.1080/15532739.2020.1703652

McNeil, J., Ellis, S. J., \& Eccles, F. J. R. (2017). Suicide in trans populations: A systematic review of prevalence and correlates. Psychology of Sexual Orientation and Gender Diversity, 4(3), 341-353. https://doi.org/10.1037/sgd0000235

Medico, D. (2019). Genres, subjectivités et corps au-delà de la binarité. Filigrane, 28(1), 57-71. https://doi.org/10.7202/1064597ar

Medico, D. (2020). Quelques considérations critiques et cliniques sur le genre et ses dissident.e.s. In Analysis, 4(3), 374-382. https://doi.org/10.1016/j.inan.2020.10.006

Moolchaem, P., Liamputtong, P., O'Halloran, P., \& Muhamad, R. (2015). The lived experiences of transgender persons: A metasynthesis. Journal of Gay and Lesbian Social Services, 27(2), 143-171. https://doi.org/10.1080/10538720.2015.1021983

Moradi, B., Tebbe, E. A., Brewster, M. E., Budge, S. L., Lenzen, A., Ege, E., Schuch, E., Arango, S., Angelone, N., Mender, E., Hiner, D. L., Huscher, K., Painter, J., \& Flores, M. J. (2016). A content analysis of literature on trans people and issues: 2002-2012. The Counseling Psychologist, 44(7), 960-995. https://doi.org/10.1177/0011000015609044 
Motmans, J., Nieder, T. O., \& Bouman, W. P. (2019). Transforming the paradigm of nonbinary transgender health: A field in transition. International Journal of Transgenderism, 20(2-3), 119-125. https://doi.org/10.1080/15532739.2019.1640514

Olson, J., Forbes, C., \& Belzer, M. (2011). Management of the transgender adolescent. Archives of Pediatrics \& Adolescent Medicine, 165(2), 171-176. https://doi.org/10.1001/archpediatrics.2010.275

Reisner, S. L., Poteat, T., Keatley, J., Cabral, M., Mothopeng, T., Dunham, E., Holland, C. E., Max, R., \& Baral, S. D. (2016). Global health burden and needs of transgender populations: A review. The Lancet, 388(10042), 412-436. https://doi.org/10.1016/S01406736(16)00684-X

Richards, C. (2020). Finitude et devenir trans. Racing the Reaper. Frontières, 37(2). https://doi.org/10.7202/1070336ar

Richards, C., Bouman, W. P., Seal, L., Barker, M. J., Nieder, T. O., \& T'SJoen, G. (2016). Non-binary or genderqueer genders. International Review of Psychiatry, 28(1), 95-102. https://doi.org/10.3109/09540261.2015.1106446

Spivey, L. A., \& Edwards-Leeper, L. (2019). Future directions in affirmative psychological interventions with transgender children and adolescents. Journal of Clinical Child \& Adolescent Psychology, 48(2), $343-356$. https://doi.org/10.1080/15374416.2018.1534207

Stephenson, R., Riley, E., Rogers, E., Suarez, N., Metheny, N., Senda, J, Saylor, K. M., \& Bauermeister, J. A. (2017). The sexual health of transgender men: A scoping review. The Journal of Sex Research, 54(4-5), $424-445$. https://doi.org/10.1080/00224499.2016.1271863

Stewart, L., O'Halloran, P., \& Oates, J. (2018). Investigating the social integration and wellbeing of transgender individuals: A metasynthesis. International Journal of Transgenderism, 19(1), 46-58. https://doi.org/10.1080/15532739.2017.1364199

Tankersley, A. P., Grafsky, E. L., Dike, J., \& Russel, T. J. (2021). Risk and resilience factors for mental health among transgender and gender nonconforming (TGNC) youth: A systematic review. Clinical Child and Family Psychology Review, 24(2), $183-206$. https://doi.org/10.1007/s10567-021-00344-6

The Lancet (2018). Gender-affirming care needed for transgender children [Editorial]. The Lancet, 391(10140), 2576. https://doi.org/10.1016/S0140-6736(18)31429-6

Turban, J. L., \& Ehrensaft, D. (2018). Research Review: Gender identity in youth: Treatment paradigms and controversies. Journal of Child Psychology and Psychiatry, 59(12), 1228-1243. https://doi.org/10.1111/jcpp.12833

Watson, R. J., \& Veale, J. (2018). Transgender youth are strong: Resilience among gender expansive youth worldwide. International Journal of Transgenderism, 19(2), 115-118. https://doi.org/10.1080/15532739.2018.1474832

White Hughto, J. M., Reisner, S. L., \& Pachankis, J. E. (2015). Transgender stigma and health: A critical review of stigma determinants, mechanisms, and interventions. Social Science \& Medicine, 147, 222-231. https://doi.org/10.1016/j.socscimed.2015.11.010

\section{Bibliographie résilience sans arbitrage : ouvrages, chapitres et rapports de recherche}

Abdellahi, É., \& Thibeault, C-A. (2021). La thérapie par les arts: un outil inspirant pour accompagner les jeunes. Dans A. Pullen Sansfaçon \& D. Medico (dir.), Jeunes trans et non binaires : de l'accompagnement à l'affirmation (pp. 306-322). Éditions RemueMénage.

Beemyn, G., \& Rankin, S. (2011). The lives of transgender people. Columbia University Press.

Ehrensaft, D. (2011). Gender born, gender made: Raising healthy gender-nonconforming children. Workman Publishing.

Espineira, K. (2021). La médiatisation des « enfants \& ados trans » : des écrans télé aux chaînes YouTube, se raconter et s'affirmer au présent. Dans A. Pullen Sansfaçon \& D. Medico (dir.), Jeunes trans et non binaires : de l'accompagnement à l'affirmation (pp. 62-77). Éditions Remue-Ménage.

Faddoul, M., \& Baril, A. (2021) Travail social et interventions transaffirmatives auprès des jeunes. Dans A. Pullen Sansfaçon \& D. Medico (dir.), Jeunes trans et non binaires : de l'accompagnement à l'affirmation (pp. 159-176). Éditions Remue-Ménage.

Frappier, A. A. (2021). Parents en transition. Dans A. Pullen Sansfaçon \& D. Medico (dir.), Jeunes trans et non binaires: de l'accompagnement à l'affirmation (pp. 233-249). Éditions Remue-Ménage.

Girard, G., Medico, D., \& Galantino, G. (2021). Santé sexuelle, vie sexuelle et amoureuse des adolescent.e.s trans. Dans A. Pullen Sansfaçon \& D. Medico (dir.), Jeunes trans et non binaires : de l'accompagnement à l'affirmation (pp. 212-233). Éditions RemueMénage.

Pullen Sansfaçon, A., \& Medico. D. (2021). Introduction : pour une approche transaffirmative. Dans A. Pullen Sansfaçon \& D. Medico (dir.), Jeunes trans et non binaires: de l'accompagnement à l'affirmation (pp. 11-40). Éditions Remue-Ménage.

Riggs, D. (2019). Working with transgender young people and their families: A critical developmental approach. Palgrave.

Robichaud, M-J., Kirichenko, V., \& Pullen Sansfaçon, A. (2021). Intervention en contexte d'autorité et de protection de la jeunesse. Dans A. Pullen Sansfaçon \& D. Medico (dir.), Jeunes trans et non binaires : de l'accompagnement à l'affirmation (pp. 177-193). Éditions Remue-Ménage. 
Sauvé, J-S., \& Pullen Sansfaçon, A. (2021). L'affirmation de l'identité des personnes mineures transgenre: une zone de turbulence éthico-juridique. Dans A. Pullen Sansfaçon \& D. Medico (dir.), Jeunes trans et non binaires : de l'accompagnement à l'affirmation (pp. 103-119). Éditions Remue-Ménage.

Susset, F., \& Rabiau, M. (2021a). Le soutien psychothérapeutiques des enfants créatif.ve.s dans leur genre, trans et non binaires. Dans A. Pullen Sansfaçon \& D. Medico (dir.), Jeunes trans et non binaires : de l'accompagnement à l'affirmation (pp. 267-286). Éditions Remue-Ménage.

Susset, F., \& Rabiau, M. (2021 b). L'accompagnement thérapeutiques des adolescent.e.s en transition. Dans A. Pullen Sansfaçon \& D. Medico (dir.), Jeunes trans et non binaires: de l'accompagnement à l'affirmation (pp. 287-305). Éditions Remue-Ménage.

Taylor, A. B., Chan, A., Hall, S. L., Pullen Sansfaçon, A., Saewyc, E. M., \& l'équipe de recherche de l'enquête canadienne sur la santé des jeunes trans (2020). Etre en sécurité, être soi-même 2019: Résultats de l'enquête canadienne sur la santé des jeunes trans et non-binaires. Stigma and Resilience Among Vulnerable Youth Centre, University of British Colombia. https://apsc-saravyc.sites.olt.ubc.ca/files/2020/03/Etre-en-Securite-Etre-Soi-Meme-2019_SARAVYC_FR.pdf

Travers, R., Bauer, G., Pyne, J., Bradley, K., Gale, L., \& Papadimitriou, M. (2012). Impacts of strong parental support for trans youth: A report prepared for children's Aid Society of Toronto and Delisle Youth Services. https://transpulseproject.ca/research/impacts-of-strong-parental-support-for-trans-youth/

Wahlen, R. (2021). Enjeux médicoéthiques posés aux médecins lors de la prescription de traitements d'affirmation de genre à des jeunes trans et non binaires. Dans A. Pullen Sansfaçon \& D. Medico (dir.), Jeunes trans et non binaires: de l'accompagnement à l'affirmation (pp. 340-360). Éditions Remue-Ménage.

Zufferey, A., \& Pullen Sansfaçon, A. (2021). Approches de groupe en méthodologie autogérée entre parents d'enfants trans. Dans A. Pullen Sansfaçon \& D. Medico (dir.), Jeunes trans et non binaires : de l'accompagnement à l'affirmation (pp. 250-263). Éditions Remue-Ménage.

Ybarra, M. L., Mitchell, K. J., \& Kosciw, J. (2015). The relation between suicidal ideation and bullying victimization in a national sample of transgender and non-transgender adolescents. Dans P. Goldblum, D. L. Espelage, J. Chu \& B. Bongar (dir.), Youth suicide and bullying: Challenges and strategies for prevention and intervention (pp. 134-145). Oxford University Press. https://doi.org/10.1093/med:psych/9780199950706.001.0001 